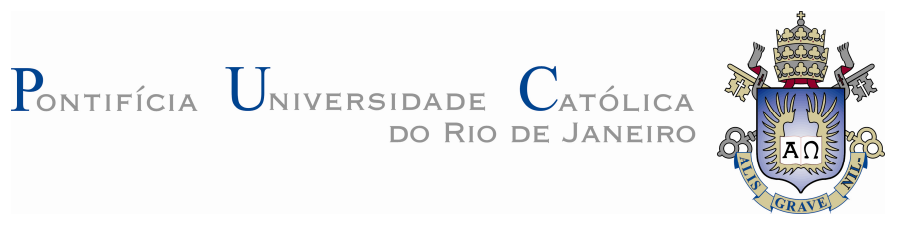

Vera Lopes de Abreu Lima

\title{
Legibilidade e leiturabilidade das bulas de medicamentos presentes no tratamento de pacientes cardíacos
}

Dissertação de Mestrado

Dissertação apresentada ao Programa de Pós-Graduação em Design da Puc-Rio como requisito parcial para obtenção do título de Mestre em Design.

Orientadora: Anamaria de Moraes 


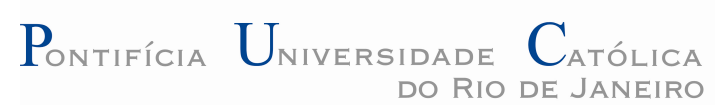

\title{
Vera Lopes de Abreu Lima
}

\section{Legibilidade e leiturabilidade das bulas de medicamentos presentes no tratamento de pacientes cardíacos}

\begin{abstract}
Dissertação apresentada ao Programa de Pós-Graduação em Design da Puc-Rio como requisito parcial para obtenção do título de Mestre em Design. Aprovada pela Comissão Examinadora abaixo assinada.
\end{abstract}

\author{
Prof.Anamaria de Moraes \\ Orientadora \\ Departamento de Artes e Design - Puc-Rio \\ Jane Dutra Sayd \\ Professora convidada \\ Departamento de Planejamento e Administração em Saúde - Uerj \\ Sydney Fernandes de Freitas \\ Professor convidado \\ Escola Superior de Desenho Industrial - Uerj \\ Professora Suplente: Valéria Rarbosa Gomes \\ Faculdade de Engenharia - Uerj \\ Prof. Paulo Fernando Carneiro de Andrade \\ Coordenador Setorial de Pós-Graduação e Pesquisa \\ do Centro de Teologia e Ciências Humanas - Puc-Rio
}


Todos os direitos reservados. É proibida a reprodução total ou parcial do trabalho sem autorização do autor, do orientador e da universidade.

Vera Lopes de Abreu Lima

Graduou-se em Desenho Industrial pela Escola Superior de Desenho Industrial - ESDI, da Universidade do Estado do Rio de Janeiro em 2000.

Ficha Catalográfica

Legibilidade e leiturabilidade das bulas de medicamentos presentes no tratamento de pacientes cardíacos / Vera Lopes de Abreu Lima ; orientador: Anamaria de Moraes. - 2007

169 f. ; $30 \mathrm{~cm}$

Dissertação (Mestrado em Artes e Design)Pontifícia Universidade Católica do Rio de Janeiro, Rio de Janeiro, 2007.

Inclui bibliografia

1. Artes - Teses. 2. Ergonomia. 3. Legibilidade e leiturabilidade. 4. Bulas de medicamento. 5. Design gráfico. 6. Informação ao paciente. I. Moraes, Anamaria. II. Pontifícia Universidade Católica do Rio de Janeiro. Departamento de Artes. III. Título.

CDD: 700 


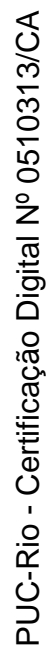

Aos meus pais,

pela oportunidade de estudar com amor. 


\section{Agradecimentos}

À professora Anamaria de Moraes, que com generosidade e competência me orientou nesta pesquisa.

Ao Dr. Raul Gonçalves Marques, pela orientação nas questões médicas e poéticas.

À Capes, pelo apoio financeiro.

Ao Instituto Nacional de Cardiologia - INC, por permitir e apoiar esta pesquisa. Agradecimento especial à Dra. Regina Maria de Aquino Xavier, Diretora Geral e ao Dr. Bernardo Rangel Dura.

Aos designers, aos médicos e aos pacientes, que não apenas se dispuseram a colaborar com esta pesquisa respondendo aos questionários e avaliações, mas também incentivaram diariamente.

A Débora, minha mãe, pela digitação.

A Marion Villas Boas, pela revisão.

A Miguel e Isabel.

Aos meus irmãos e aos meus amigos, especialmente Marcelo Lins, Suzana Ichigo, Flávia Soares, David Amiel, Ana Casas e Francesco Venturin, pela companhia inteligente.

A Alita.

À professora Edna Cunha Lima, pelo incentivo.

À Puc-Rio, em particular:

ao Departamento de Artes;

ao Setor de Bibliotecas;

ao Departamento de Letras;

ao Departamento de Educação, pricipalmente a Leandro Konder, Rosália Duarte, Fernando e Rita pelas ótimas tardes de sexta-feira.

Ao meu pai, Henrique de Abreu Lima, pela ajuda essencial em todas as traduções. 


\section{Resumo}

Lima, Vera Lopes de Abreu; de Moraes, Anamaria. Legibilidade e leiturabilidade das bulas de medicamentos presentes no tratamento de pacientes cardíacos. Rio de Janeiro, 2007, 169p. Dissertação de Mestrado - Departamento de Artes e Design, Pontifícia Universidade Católica do Rio de Janeiro.

Com o envelhecimento da população brasileira o número de pacientes de doenças crônicas aumenta acentuadamente. A informação adequada ao paciente é elemento fundamental para que este seja parte ativa, aumentando assim a eficiência do tratamento. As bulas dos medicamentos podem contribuir de forma significativa para a informação dos pacientes. Entretanto, para que isto ocorra, é necessário que a bula seja projetada levando em consideração as necessidades do usuário. A hipótese desta pesquisa é que a desconsideração do usuário no projeto das bulas presentes no tratamento de doenças cardiovasculares acarreta dificuldade na legibilidade e leiturabilidade destas, gerando riscos pelo uso inadequado do medicamento. Esta pesquisa tem o objetivo de colaborar para o aumento da segurança dos pacientes crônicos na administração de medicamentos, através da identificação dos fatores que prejudicam a legibilidade e leiturabilidade das bulas. Para se alcançar estes objetivos foram utilizados métodos e técnicas em duas etapas. Primeiramente, foram realizadas entrevistas com médicos e pacientes, e avaliações heurísticas de duas bulas de um medicamento antihipertensivo largamente usado. Em seguida, foram realizados questionários junto a médicos e pacientes do Instituto Nacional de Cardiologia, no Rio de Janeiro, a respeito da legibilidade e leiturabilidade das bulas, assim como sua colaboração na compreensão da enfermidade e tratamento. Através destes métodos, foram confirmados problemas graves na legibilidade e leiturabilidade das bulas presentes no tratamento de doentes cardíacos, gerando insegurança do paciente e perigo no uso de medicamentos.

\section{Palavras-chave}

Ergonomia, legibilidade e leiturabilidade, bulas de medicamento, design gráfico, informação ao paciente. 


\section{Abstract}

Lima, Vera Lopes de Abreu; de Moraes, Anamaria. Legibility and Readability of medicines package inserts present on cardiovascular patients'treatment. Rio de Janeiro, 2007. 169p. MSc. Dissertation Departamento de Artes e Design, Pontifícia Universidade Católica do Rio de Janeiro.

Due the increasing aging of Brazilian population, it is also increasing the number of chronic diseases' patients. Adequate information is vital for the patient to take an active role on treatment, increasing treatments' efficiency. Patient packages inserts may contribute significantly for patients' information. This research is based on the hypotheses that there is a lack on users' needs during the patient leaflets design. This lack may cause legibility and readability problems and, consequently, risks by the inadequate use of medicine. The aim of the present research is to contribute to the safe use of medicines from cardiovascular patients. Factors that may prejudice leaflets'legibility and readability were investigated. The research techniques used were: Firstly interview with physicians and patients, and heuristic evaluation of two leaflets of the most widely sold anti hypertensive medicine in Brazil. Then, two questionnaires were applied; one with physicians and the other with patients of the Instituto Nacional de Cardiologia (National Institute of Cardiology), in Rio de Janeiro. Those two questionnaires concerned on the leaflets' legibility and readability, and its cooperation on the patients' comprehension of their disease and treatment. Serious legibility and readability problems were identified on the leaflets, generating patient insecurity and unsafe medicines'use.

\section{Key-words}

Ergonomics, legibility and readability, medicine leaflets, graphic design, information to the patient. 


\section{Sumário}

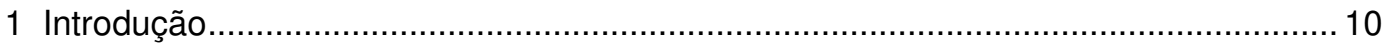

2 O aumento de doenças crônicas

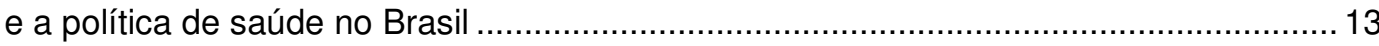

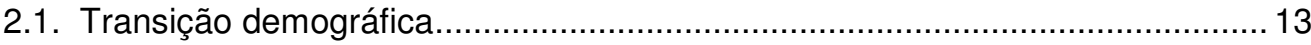

2.2. Transição epidemiológica ....................................................................... 16

2.2.1. O aumento dos custos com saúde

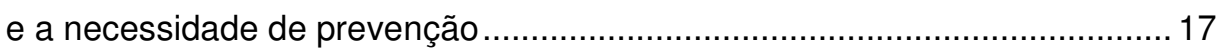

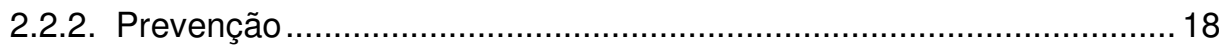

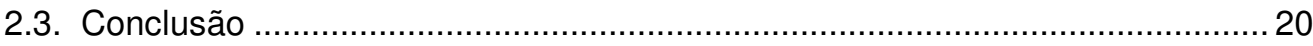

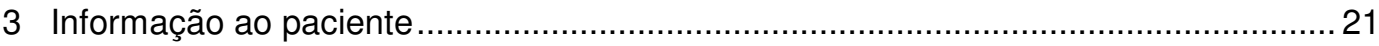

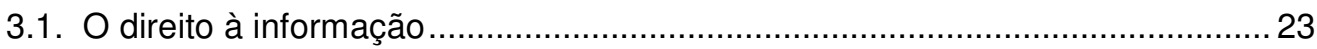

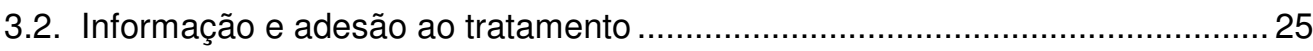

3.2.1. Informação: elemento-chave no tratamento de doenças crônicas....... 27

3.2.2. Médico como fonte de informação verbal ao paciente .......................... 29

3.2.3. A bula como fonte de informação escrita ao paciente........................... 32

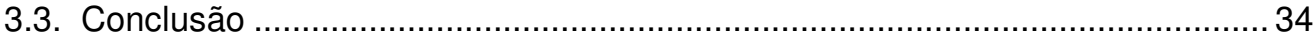

4 Uma bula que leve em conta o usuário:

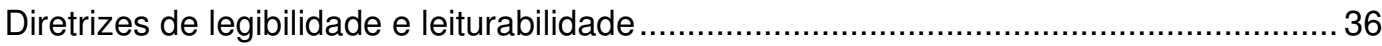

4.1. Leiturabilidade e legibilidade: definição de conceitos ..........................................36

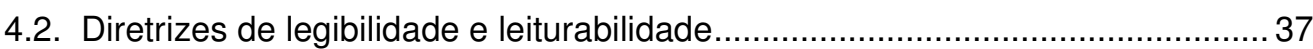

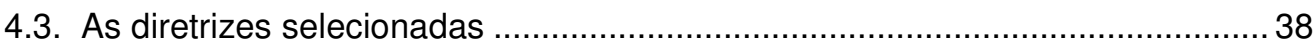

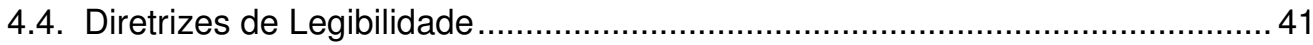

4.4.1. Guia de design e layout em linguagem simples ................................ 41

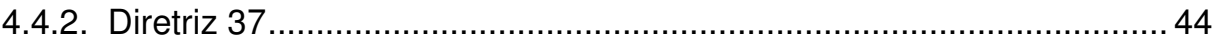

4.4.3. Como escrever informações médicas em linguagem simples ............ 45

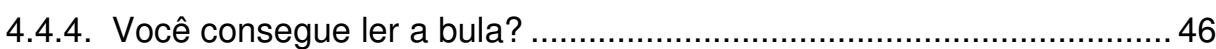

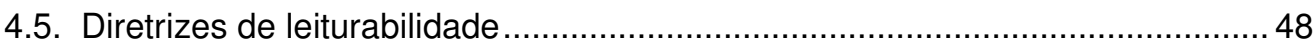

4.5.1. Diretrizes de comunicação

de riscos e benefícios nas bulas para o paciente............................................ 48

4.5.2. Como escrever informação médica em linguagem simples ..................54

4.5.3. Você consegue ler a bula? ........................................................... 56

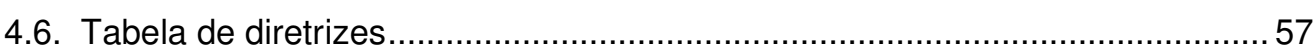

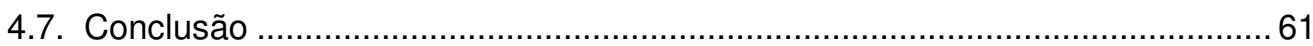

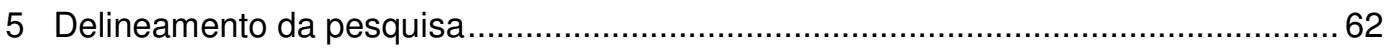

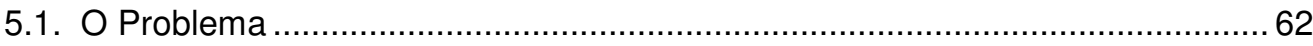

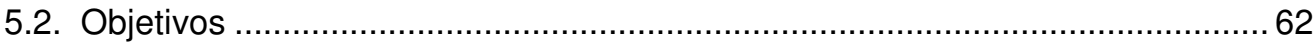

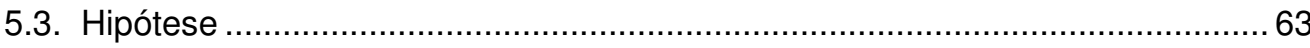




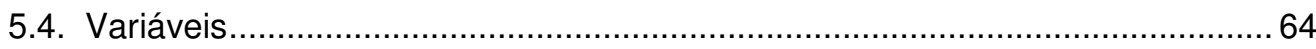

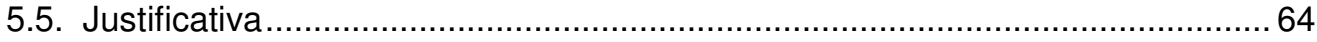

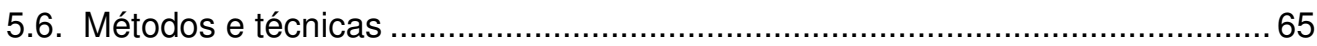

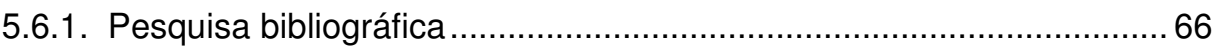

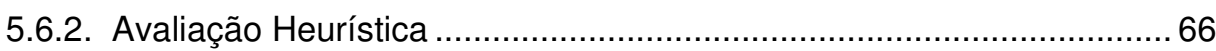

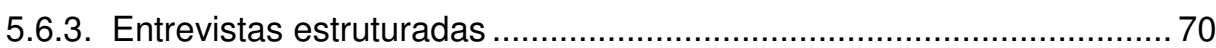

5.6.4. Questionários e formulários .......................................................... 70

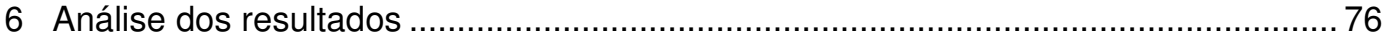

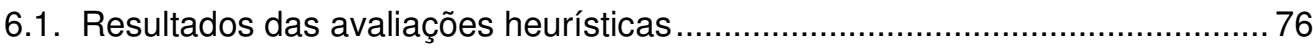

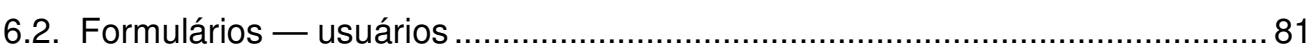

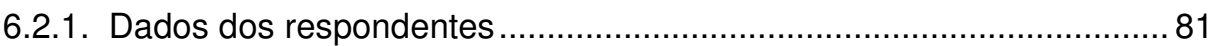

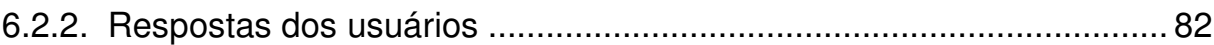

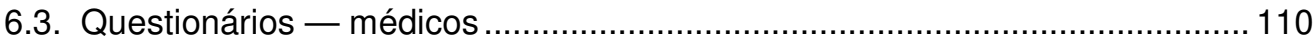

6.3.1. Dados gerais dos respondentes ...................................................... 110

6.3.2. A bula nas seguintes relações:...................................................... 112

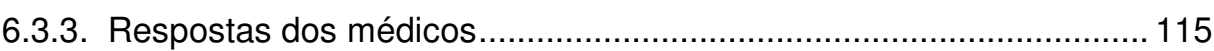

6.3.4. Comparação respostas pacientes e médicos..................................... 128

7

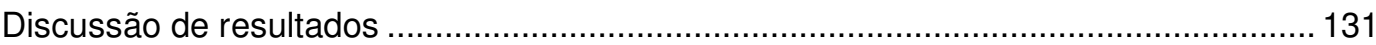

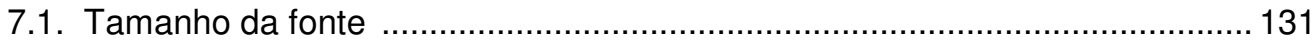

7.2. Outros problemas de legibilidade ............................................................. 133

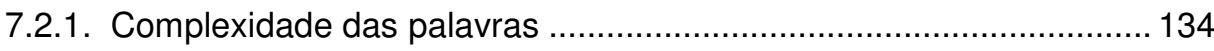

7.2.2. Deve haver uma seção de informações-chave ................................. 135

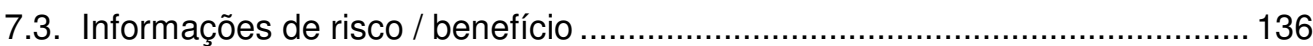

7.4. A atribuição da culpa dos problemas de legibilidade e leiturabilidade ao usuário ........................................................ 136

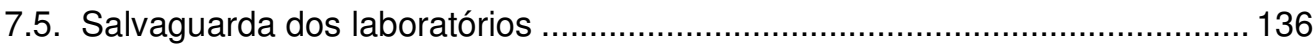

7.6. A resistência médica em informar

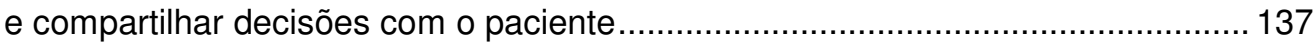

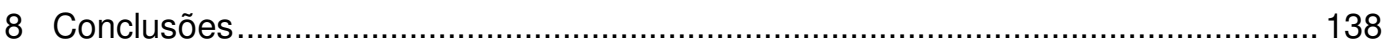

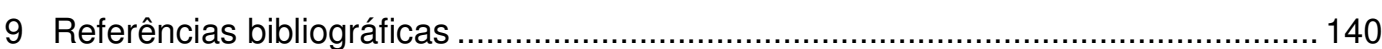

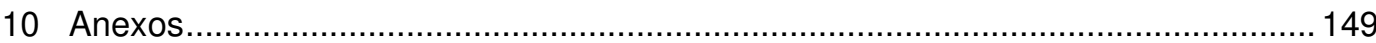

